\title{
Centaurus A as a source of extragalactic cosmic rays with arrival energies well beyond the GZK cutoff
}

\author{
Gustavo E. Romero ${ }^{1,2}$, Jorge A. Combi ${ }^{1}$, \\ Santiago E. Perez Bergliaffa ${ }^{2}$, Luis A. Anchordoqui ${ }^{2}$ \\ ${ }^{1}$ Instituto Argentino de Radioastronomía, C.C. 5, 1894 Villa Elisa, Argentina \\ ${ }^{2}$ Laboratorio de Física Teórica, Departamento de Física, U.N.L.P., C.C. 67, \\ 1900 La Plata, Argentina
}

\begin{abstract}
The ultra-high energy cosmic rays recently detected by several air shower experiments could have an extragalactic origin. In this case, the nearest active galaxy Centaurus A might be the source of the most energetic particles ever detected on Earth. We have used recent radio observations in order to estimate the arrival energy of the protons accelerated by strong shock fronts in the outer parts of this southern radio source. We expect detections corresponding to particles with energies up to $\sim 2.2 \times 10^{21} \mathrm{eV}$ and an arrival direction of $\left(l \approx 310^{\circ}, b \approx 20^{\circ}\right)$ in galactic coordinates. The future Southern Hemisphere Pierre Auger Observatory might provide a decisive test for extragalactic models of the origin of the ultra-high energy cosmic rays.
\end{abstract}

Keywords: UHE cosmic rays, active galaxies: Cen A, acceleration mechanisms, propagation effects.

PACS numbers: 96.40 98.70.S 95.85.R $13.85 . \mathrm{T}$ 


\section{Introduction}

The origin of cosmic rays (CR) detected in the neighborhood of Earth is still an unsettled issue. Most of the low energy particles are believed to be produced by supernova explosions in the galactic interstellar medium [1]. Higher energy CR could be accelerated by stellar explosions in strong stellar winds [2]. The basic acceleration mechanism in all these models is diffuse shock acceleration [3]. Different observational facts, such as a break in the CR spectrum at $\sim 5 \times 10^{18}$ $\mathrm{eV}$, the change in the composition of the rays from heavy nuclei below this break to light nuclei and protons at higher energies, and the arrival directions of the highest energetic particles seem to support the idea that the component with energies below the break is predominantly galactic whilst the particles with energies above $10^{19} \mathrm{eV}$ have an extragalactic origin. The hot spots of extended radio galaxies (i.e. the end-points of powerful jets ejected by the central active galactic nucleus) have been suggested as the dominant source of ultra-high energy CR «1 In such a case, particles are assumed to be accelerated by first order Fermi mechanism through the strong, nonrelativistic shock waves generated by huge plasma collisions.

When the ultra-high energy protons are injected in the intergalactic medium, traveling losses originated by interactions with the $2.73 \mathrm{~K}$ cosmic background radiation are expected to produce a high energy cutoff in the particle spectrum at energies $\sim 10^{20} \mathrm{eV}$, according to the predictions of Greisen [5] and Zatsepin and Kuźmin [6] (the so-called "GZK cutoff"). Nevertheless, recent detections with the Akeno Giant Air Shower Array [7] and the Utah Fly's Eye detector [8, 9] have revealed the existence of $\mathrm{CR}$ with energies above the mentioned cutoff. A detailed analysis of the arrival directions of these and other energetic events has shown a correlation with the general direction of the supergalactic plane, where many potential extragalactic sources of CR are located [10]. Despite these correlations, there seems to be no conclusive evidence for an extragalactic origin of the most energetic CR.

In this paper we present a concrete candidate for testing the acceleration models that involve hot spots and strong shock waves as the source of the ultra-high energy component of the CR. Using polarization measurements and synchrotron emission as a trace, we can make an estimate of the maximum energy of the protons injected in the extragalactic medium and the corresponding arrival energy for the case of the nearest active galaxy: the southern radio source Centaurus A (Cen A)円. If the basic model is correct, a southern hemisphere detector should

\footnotetext{
${ }^{1}$ Cen A was suggested as a possible source of ultra-high energy cosmic rays by G. Cavallo [11, from quite general energetic arguments.
} 
register events produced by particles with energies beyond $10^{21} \mathrm{eV}$ as we shall see. But first, let us briefly discuss some physical features of Cen A.

\section{Cen $\mathrm{A}$ and $\mathrm{CR}$ acceleration}

Cen $\mathrm{A}$ is a complex and extremely powerful radio source identified at optical frequencies with the galaxy NGC 5128. It is the nearest radio galaxy, at a distance of $\sim 3.5 \mathrm{Mpc}$ according to recent works on globular clusters [12] and planetary nebulae [13]. Radio observations at different wavelengths [14, 15] show a structure composed by a compact core, a one-sided jet, Double Inner Lobes, a Northern Middle Lobe, and two Giant Outer Lobes. This morphology, together with the polarization data obtained by Junkes et al. [15] and the large-scale radio spectral index distribution computed by two of us [16], strongly support the picture of an active radio galaxy with a jet forming a relatively small angle with the line of sight. The jet would be responsible for the formation of the Northern Inner and Middle Lobes when interacting with the interstellar and intergalactic medium, respectively. The Northern Middle Lobe can be interpreted as a "working surface" [17] at the end of the jet, a place where strong shocks are produced by plasma collisions, i.e. it can be considered as the hot spot of a galaxy with a peculiar orientation.

The acceleration of particles in the hot spot is the result of repeated scattering back and forth across a strong shock front in a partially turbulent magnetic field. This process has been studied in detail by Biermann and Strittmatter [18. Assuming that the energy density per unit of wave number of MHD turbulence is of Kolmogorov type, i.e. $I(k) \alpha k^{-s}$ with $s=5 / 3$, we have an acceleration time scale for protons given by:

$$
E_{p}\left(\frac{d E_{p}}{d t}\right)_{\mathrm{ACC}}^{-1} \approx \frac{40}{\pi c} \beta_{\mathrm{JET}}^{-2} u R_{\mathrm{HS}}^{2 / 3}\left(\frac{E_{p}}{e B}\right)
$$

where $\beta_{\text {JET }}$ is the jet velocity in units of $c, u$ is the ratio of turbulent to ambient magnetic

energy density in the hot spot (of radius $R_{\mathrm{HS}}$ ), and $B$ is the total magnetic field strength. The acceleration process will be efficient as long as the energy losses by synchrotron radiation and photon-proton interactions do not become dominant. Considering an average cross section $\bar{\sigma}_{\gamma p}$ for the three dominant pion-producing interactions [19]:

$$
\begin{gathered}
\gamma+p \rightarrow p+\pi^{0} \\
\gamma+p \rightarrow n+\pi^{+} \\
\gamma+p \rightarrow p+\pi^{+}+\pi^{-},
\end{gathered}
$$


we get the time scale of the energy losses within a certainty of $80 \%$ :

$$
E_{p}\left(\frac{d E_{p}}{d t}\right)_{\text {LOSS }}^{-1} \approx \frac{6 \pi m_{p}^{4} c^{3}}{\sigma_{\mathrm{T}} m_{e}^{2} B^{2}(1+a A)} E_{p}^{-1}
$$

where $a$ stands for the ratio of photon to magnetic energy densities, $\sigma_{T}$ is the classical Thomson cross section, and $A$ gives a measure of the relative strength of $\gamma p$ interactions against the synchrotron emission. Biermann and Strittmatter [18] have estimated $A \approx 200$, almost independently of the source parameters. The most energetic protons injected in the intergalactic medium will have an energy that can be obtained by balancing the energy gains and losses:

$$
E_{p, \max }=1259.3 c e^{1 / 4} \beta_{\mathrm{JET}}^{3 / 2}\left(\frac{u}{\sigma_{\mathrm{T}}}\right)^{3 / 4} \frac{m_{p}^{2}}{m_{e}^{3 / 2}} R_{\mathrm{HS}}^{-1 / 2} B^{-5 / 4}(1+A a)^{-3 / 4} \mathrm{MeV}
$$

The acceleration model also predicts a power-law spectrum for the number of particles per unit of energy: $N(E) \propto E^{-p},\left(E<E_{p, \max }\right)$.

In the case of Cen $\mathrm{A}$, we can estimate $u$ from the radio spectral index of the synchrotron emission in the Northern Middle Lobe and the observed degree of linear polarization in the same region. We get $u \approx 0.4$. The size of the hot spot can be directly measured from the large-scale map obtained by Junkes et al. [15] with the assumed distance of $3.5 \mathrm{Mpc}$, giving as a result $R_{\mathrm{HS}} \approx 1.75 \mathrm{kpc}$.

The equipartition magnetic field can be obtained for the pre-shock region from the detailed radio observations by Burns et al [14]. The field component parallel to the shock will be amplified in the post-shock region by a compression factor $\xi$. In the case of a strong, nonrelativistic shock front, $\xi \rightarrow 4$ [20], and then, if $B_{\|} \sim B_{\perp}$, we have:

$$
\begin{aligned}
B & \approx\left(\xi^{1 / 2}+1\right)^{1 / 2} B_{\perp} \\
& \approx 5 \times 10^{-5} \mathrm{G}
\end{aligned}
$$

An enhancement of the $B_{\|}$component in the Northern Middle Lobe can be clearly seen in the polarization maps displayed in ref. [15]. The value of $\beta_{\text {JET }}$ is uncertain. We shall assume $\beta_{\mathrm{JET}} \approx 0.3$, a typical value for similar sources 21 .

With the above mentioned values for the input parameters in Eq. (3), the maximum energy of the protons injected in the intergalactic space results

$$
E_{p, \max } \approx 2.7 \times 10^{21} \mathrm{eV}
$$

We can infer the index in the power-law spectrum from multifrequency observations of the synchroton radiation produced by the leptonic component of the particles accelerated in the 
source (see the standard formulae, for instance, in the book by Pacholczyk [22]). Using the radio spectral index obtained by Combi and Romero for the hot spot region [16], we get $p=2.2$.

\section{Propagation effects}

In this section we shall briefly discuss the proton energy losses due to collisions with microwave photons during their travel to Earth, and the modifications in the energy spectrum arised in these interactions. The losses can be estimated as:

$$
\beta\left(E_{p}\right)=-\frac{1}{E_{p}} \frac{d E_{p}}{d \tau}=\frac{-k T}{2 \pi^{2} \hbar^{3} c^{2} \gamma_{p}^{2}} \sum_{j} \int_{\epsilon_{j, \mathrm{th}}}^{\infty} \epsilon f_{j}(\epsilon) \ln \left[1-\exp \left(\frac{-\epsilon}{2 \gamma_{p} k T}\right)\right] d \epsilon
$$

where $T=2.73 \mathrm{~K}, \tau$ is the traveling time, $f_{j}(\epsilon)$ is the cross section weighted by inelasticity of the $j$ th reaction channel, and the sum is carried out over all channels. The integration is over the photon energy in the proton rest frame, from the threshold. For energies higher than $3 \times 10^{20} \mathrm{eV}$, and taking into account just the dominant pion-producing interactions, this equation becomes [23]:

$$
-\frac{1}{E_{p}} \frac{d E_{p}}{d \tau} \approx 1.8 \times 10^{-8} \mathrm{yr}^{-1}
$$

According to this, the losses are almost neglectable due to the proximity of the source, yielding a maximum arrival energy $E_{p, \text { max }}^{\text {arr }} \approx 2.2 \times 10^{21} \mathrm{eV}$. The arrival direction of these $\mathrm{CR}$ will be approximately $\left(l \approx 310^{\circ}, b \approx 20^{\circ}\right)$, in galactic coordinates. Intergalactic and galactic magnetic fields with strength of the order of $\mathrm{nG}$ and $\mu \mathrm{G}$, respectively, cause no relevant deviations from the line of sight, due to the distances and energies involved in the case under consideration. However, a spreading in the arrival direction pattern should be observed for a relatively large number of low energy events coming from Cen A.

Both the $\gamma \pi$ energy losses and the conservation of the total number of protons in the spectrum are expected to produce only a slight modification of the original energy spectrum. This modification can be quantitatively described in terms of a factor given by

$$
\eta\left(E_{p}, \tau\right)=\frac{N\left(E_{p}, \tau\right)}{N\left(E_{p}, 0\right)}
$$

In the case of a nearby source, an analitical expression of $\eta\left(E_{p}, \tau\right)$ has been found by Berezinsky and Grigor'eva [23]:

$$
\eta\left(E_{p}, \tau\right)=1+\beta\left(E_{p}\right) \tau\left(\frac{d \ln \beta\left(E_{p}\right)}{d \ln E_{p}}-p+1\right)
$$


At very high energies $\left(E_{p}>5 \times 10^{20}\right), d \ln \beta\left(E_{p}\right) / d \ln E_{p} \rightarrow 0$, and then $\eta\left(E_{p}, \tau\right) \rightarrow 1-(p-1) \tau \omega_{0}$, where $\omega_{0} \approx 1.8 \times 10^{-8} \mathrm{yr}^{-1}$. Since $\eta<1$, there will be a slight fall in the spectrum, as we already

mentioned in the case of the highest energy protons. At lower energies $\left(E_{p} \lesssim 3 \times 10^{20} \mathrm{eV}\right)$ the modification factor is

$$
\eta\left(E_{p}, \tau\right) \approx 1+\left(\frac{\tau}{\tau_{\pi}}\right) e^{-\left(\epsilon_{\pi} / E_{p}\right)}\left(\frac{\epsilon_{\pi}}{E_{p}}-p+1\right)
$$

where $\epsilon_{\pi} \approx 3 \times 10^{20} \mathrm{eV}$, and $\tau_{\pi} \approx 1.7 \tau$. This will produce a small bump in the spectrum. In brief, the overall spectrum will be scarcely modified.

An additional interesting consequence of the model is that the $\gamma+p \rightarrow p+\pi^{0}$ interactions would be a source of $\gamma$-rays through $\pi^{0}$ decay $\left(\pi^{0} \rightarrow 2 \gamma\right)$. Considering a power-law dependence for the isotropic and homogeneous photon distribution in the source [18]:

$$
N_{\gamma}(\epsilon)=n_{0}\left(\frac{\epsilon}{\epsilon_{0}}\right)^{-2} \quad \epsilon_{0} \leq \epsilon \leq \epsilon_{\max }
$$

we can compute the energy range of the total pion spectrum [24]. Using $\gamma_{p, \max } \approx 10^{12}$ and $\epsilon_{\max } \approx 1 \mathrm{MeV}$, we obtain (in the observer's frame):

$$
E_{\pi} \in\left[4 \times 10^{10} \mathrm{eV}, 5 \times 10^{20} \mathrm{eV}\right]
$$

If the proton distribution $N\left(E_{p}\right)$ has the same index as the electron distribution $N\left(E_{e}\right)$, as we have assumed, the pion distribution function will be

$$
N\left(E_{\pi}\right) \propto E_{\pi}^{-\frac{p+1}{2}}
$$

In the case of Cen $\mathrm{A}$, we have $N\left(E_{\pi}\right) \propto\left(\gamma_{\pi} m_{\pi} c^{2}\right)^{-1.6}$.

Emission at low $\gamma$-ray frequencies from Cen A has been detected by the Compton Gamma Ray Observatory [25], and by experiments with balloons [26]. However, these $\gamma$-rays could not be produced by $\pi^{0}$ decays in the acceleration region because, according to (11), the relevant energy ranges from $\sim 20 \mathrm{GeV}$ up to a value comparable to those of ultra-high energy $\mathrm{CR}$. It might be worthwhile then to perform observations of Cen A with instruments sensitive to TeV energies, for instance with the new Imaging Atmospheric Cerenkov Telescopes [27.

\section{Final comment}

We expect the detection of an extensive air shower produced by the collision of protons with energies $E_{p}>100 \mathrm{EeV}$ with the Earth's atmosphere. Since the arrival direction of these extremely energetic particles is well known, the future Hybrid Pierre Auger Observatory [28, 29] 
will provide a decisive test for the models that invoke an extragalactic origin for the ultra-high energy component of the cosmic rays.

Remarks by Alan Watson are gratefully acknowledged. This work has been partially supported by CONICET and UNLP.

\section{References}

[1] R. D. Blandford and J. P. Ostriker, Ap.J. 237, 793 (1980).

[2] H. J. Völk and P. L. Biermann, Ap. J. Letters 333, L65 (1988).

[3] L. O'C. Drury, Rep. Prog. Phys. 46, 973 (1983).

[4] J. P. Rachen and P. L. Biermann, Astron. Astrophys. 272, 161 (1993).

[5] K. Greisen, Phys. Rev. Lett. 16, 748 (1966).

[6] G. T. Zatsepin and V. A. Kuźmin, Pis'ma Zh. Eksp. Teor. Fiz. 4, 114 (1966).

[7] N. Hayashida, et al., Phys. Rev. Lett. 73, 3491 (1994).

[8] D. J. Bird, et al., Phys. Rev. Lett. 71, 3401 (1993).

[9] D. J. Bird, et al., Ap. J. 441, 144 (1995).

[10] T. Stanev, P. L. Biermann, J. Lloyd-Evans, J. P. Rachen and A. A. Watson, Phys. Rev. Lett., 73, 3056 (1995).

[11] G. Cavallo, Astron. Astrophys. 269, 45 (1978).

[12] G. L. H. Harris, et al., Ap. J. 287, 175 (1984).

[13] G. H. Jacobi, R. Ciardullo and H. Ciford, in The Extragalactic Distance Scale, ASP Conf. Ser. 4, San Francisco, p.42 (1988).

[14] J. O. Burns, E. D. Feigelson and E. J. Schreier, Ap. J. 273, 128 (1983).

[15] N. Junkes, et al., Astron. Astrophys. 269, 29 (1993).

[16] J. A. Combi and G. E. Romero, Astron. Astrophys., submitted. 
[17] R. D. Blandford and M. J. Rees, Monthly Not. Royal Astron. Soc. 169, 395 (1974).

[18] P. L. Biermann and P. A. Strittmatter, Ap. J. 322, 643 (1987).

[19] T. A. Armstrong, et al., Phys. Rev. D 5, 1640 (1972).

[20] L. Landau and E. Lifchitz, Fluid Mechanics (Pergamon Press, Oxford, 1958).

[21] K. Meisenheimer, et al., Astron. Astrophys. 219, 63 (1989).

[22] A. G. Pacholczyk, Radio Astrophysics (Freeman, San Francisco, 1970).

[23] V. S. Berezinsky and S. I. Grigor'eva, Astron. Astrophys. 199, 1 (1988).

[24] K. Mannheim and P. L. Biermann, Astron. Astrophys. 221, 211 (1989).

[25] R. L. Kinzer, et al., Ap. J. 449, 105 (1995).

[26] P. von Ballmoos, R. Diehl and V. Schünfelder, Ap. J. 312, 134 (1987).

[27] F.A. Aharonian, Nucl. Phys. B (Proc. Suppl.) 39A, 193 (1995).

[28] J. W. Cronin, The Highest Energy Particles Produced in the Universe: Cosmic Rays, talk delivered at the Auger Project Workshop, Bariloche, Argentina, October 1995.

[29] A. A. Watson, The Highest Energy Cosmic Rays: Recent Measurements and their Instrumentation, talk delivered at the Auger Project Workshop, Bariloche, Argentina, October 1995. 